\title{
Associação entre o autocuidado e a qualidade de vida de pacientes com doença renal crônica
}

Loren Caroline Bettoni ${ }^{1}$, Ana Carolina Ottaviani ${ }^{2}$, Fabiana Souza Orlandi ${ }^{3}$

\footnotetext{
${ }^{1}$ Discente do curso de Graduação em Gerontologia da Universidade Federal de São Carlos. São Carlos, SP, Brasil. E-mail: loren.bett@hotmail.com.

${ }^{2}$ Gerontóloga, Mestre em Enfermagem. Discente do Programa de Pós-Graduação em Enfermagem, nível Doutorado, da Universidade Federal de São Carlos. São Carlos, SP, Brasil. E-mail: anacarolina_ottaviani@hotmail.com.br.

${ }^{3}$ Enfermeira, Doutora em Ciências da Saúde. Professora Adjunta da Universidade Federal de São Carlos. São Carlos, SP, Brasil. E-mail: fabi_ferreira@yahoo.com.br.
}

Recebido: 16/10/2016.

Aceito: 20/03/2017.

Publicado: 29/05/2017.

Como citar esse artigo:

Bettoni LC, Ottaviani AC, Orlandi FS.

Associação entre o autocuidado e a qualidade de vida de pacientes com doença renal crônica. Rev. Eletr. Enf. [Internet]. 2017 [acesso em:

____19:a14. Disponível em: http://dx.doi.org/10.5216/ree.v19.27442.

\section{RESUMO}

Este estudo avaliou a capacidade para o autocuidado e a associação com a qualidade de vida de pessoas com Doença Renal Crônica. Pesquisa correlacional, de corte transversal. A amostra foi composta por 100 indivíduos em tratamento hemodialítico de uma unidade de terapia renal substitutiva do interior do estado de São Paulo. Os dados foram coletados por meio de entrevista individual, utilizando-se um questionário para caracterização sociodemográfica, a Escala de Avaliação do Agenciamento de Autocuidado Revisada (ASAS-R) e Kidney Disease and Quality of Life Short - Form (KDQOL-SF). O escore médio da ASAS-R foi de $60,64( \pm 8,24)$ indicando boa capacidade para autocuidado. Verificou-se relação positiva, de moderada magnitude e estatisticamente significante entre a capacidade para o autocuidado e os domínios do KDQOL-SF: sintomas/problemas, função cognitiva, funcionamento físico, bem-estar emocional e energia/fadiga. Concluise que o autocuidado está relacionado com a qualidade de vida nos pacientes com doença renal crônica em hemodiálise.

Descritores: Autocuidado; Qualidade de Vida; Diálise Renal; Insuficiência Renal Crônica.

\section{INTRODUÇÃO}

As Doenças Crônicas Não Transmissíveis (DCNTs) são consideradas um crescente problema de saúde pública, com altos índices de mortalidade e incapacidades em todo o mundo ${ }^{(1)}$. Dentre as DCNTs, tem-se a Doença Renal Crônica (DRC), caracterizada por anormalidade da estrutura e/ou função dos rins presentes por mais de três meses com implicação para a saúde ${ }^{(2)}$. De acordo com o censo da Sociedade Brasileira de Nefrologia estima-se que no ano de 2011 cerca de 91.300 pacientes estavam em diálise no Brasil, e o número estimado de pacientes que iniciaram o tratamento no ano de 2010 foi de 18.972. A maioria dos pacientes 
com DRC em terapia de substituição renal, realizam hemodiálise $(91,6 \%)$ e apenas $8,4 \%$ a diálise peritoneal ${ }^{(3)}$.

A hemodiálise é um tratamento que interfere no cotidiano do indivíduo, impondo limitações que afetam aspectos biológicos, psicológicos e sociais. Com isso, há uma ruptura do estilo de vida anterior, fazendo com que haja necessidade de adaptação à nova condição, na qual o paciente se encontra ${ }^{(4-5)}$. É necessário que a família, e a equipe de saúde, busquem estimular as capacidades, as habilidades e o potencial de cada indivíduo, a fim de que ocorra a adequação ao tratamento e ao novo estilo de vida ${ }^{(5)}$.

As condições crônicas, sobretudo a DRC requer do indivíduo o gerenciamento do autocuidado contínuo e permanente, além de mudanças no estilo de vida e no comportamento, para a prevenção de agravos e melhoria da qualidade de vida ${ }^{(1)}$. O fato de a DRC ser uma patologia que demanda uma maior disciplina, leva à necessidade de ações de autocuidado, a fim de se obter uma melhor qualidade de vida ${ }^{(6)}$.

O autocuidado tem sido uma alternativa encontrada com o propósito de estimular os indivíduos com diagnósticos de doenças crônicas, a participar ativamente do tratamento, contribuindo para o aumento de suas responsabilidades nos resultados de assistência ${ }^{(7)}$.

O presente estudo considera o autocuidado como a "capacidade que o indivíduo tem de desempenhar ou praticar atividades em seu próprio benefício, a fim de manter a vida, a saúde e o bem-estar” ${ }^{(8)}$. Admite-se que o indivíduo é competente para exercer o autocuidado, quando o mesmo possui a capacidade para diferenciar fatores que devem ser controlados, decidir o que pode e deve ser feito, planejar o tratamento terapêutico e desempenhar ações com o seu próprio cuidado ${ }^{(8)}$.

O paciente com DRC que inicia o tratamento hemodialítico é submetido a mudanças no seu cotidiano. Todas as limitações resultam em problemas, como: ansiedade prévia e durante o tratamento, perda significativa de autonomia, dificuldade em lidar com a doença, falta de apoio familiar que podem prejudicar a saúde psicológica e física ${ }^{(9)}$. Por isso estimular as ações de cuidado com si mesmo nos indivíduos com DRC, favorece a manutenção da autonomia e da qualidade de vida durante todo o tratamento hemodialítico ${ }^{(10)}$.

A Organização Mundial da Saúde (OMS) considera a Qualidade de Vida Relacionada à Saúde (QVRS) um fator importante, que aborda os aspectos relacionados à percepção da saúde, funções físicas, sociais e psicológicas, sendo um importante indicador de saúde e bem-estar dos pacientes com DRC ${ }^{(11)}$.

Considerando o impacto negativo da qualidade de vida sobre as ações de autocuidado, acredita-se que este estudo contribua para a elaboração de estratégias de melhorias e manutenção da assistência prestada aos pacientes com DRC, buscando estimular ações de autocuidado, e obter maior aderência e participação dos pacientes e de suas famílias no processo terapêutico ${ }^{(12)}$.

Ressalta-se a importância do papel da enfermagem, como integrante da equipe de saúde na promoção do restabelecimento da saúde do paciente, bem como, a implementação de práticas assistenciais e educativas, que busquem trazer não só ao paciente, mas a família, orientações que valorizem o ato de realizar e manter ações e atividades para o autocuidado ${ }^{(13)}$.

Diante do exposto, o estudo teve por objetivo avaliar a capacidade para o autocuidado de pessoas com doença renal crônica em hemodiálise e verificar sua relação com a qualidade de vida. 


\section{MÉTODO}

Trata-se de um estudo correlacional, de corte transversal, com abordagem quantitativa, desenvolvido em uma unidade de terapia renal substitutiva do interior do estado de São Paulo.

A amostra foi selecionada a partir de 165 indivíduos atendidos pela unidade de terapia renal substitutiva no período da coleta. Destes, 145 realizavam o tratamento por hemodiálise e 20, por diálise peritoneal. Foram convidados a participar da pesquisa todos os indivíduos em tratamento hemodialítico, porém houve recusas, falecimentos e em alguns casos os pacientes não apresentavam bom estado clínico. Assim, a amostra foi composta por 100 participantes que atenderam aos critérios de elegibilidade: aceitar participar da pesquisa, ter idade igual ou superior a 18 anos, com diagnóstico de DRC em estágio terminal, ou seja, em tratamento hemodialítico. Com a concordância em participar da pesquisa, todos assinaram o Termo de Consentimento Livre e Esclarecido (TCLE).

Para a coleta de dados foi utilizado um questionário de caracterização sociodemográfica, a Escala de Avaliação do Agenciamento de Autocuidado Revisada (ASAS-R) e o Kidney Disease and Quality of Life Short Form (KDQOL-SF).

A Escala de Avaliação da Capacidade para o Autocuidado (ASAS) foi desenvolvida por um grupo de estudiosos americanos e holandeses, pertencentes ao Conference Group in Nursing Development (NDCG), com 24 itens e cinco opções de respostas do tipo Likert, com intuito de verificar os comportamentos promotores de saúde ${ }^{(14)}$. A identificação de ajustes nessa escala foi apontada e analisada resultando na ASASR, com a exclusão de nove itens e a descrição de três fatores (1- Ter capacidade para o autocuidado, 2Desenvolvimento para a capacidade de autocuidado e 3 - Falta de capacidade para o autocuidado ${ }^{(15)}$. Foi traduzida, adaptada e validada para o contexto brasileiro, em portadores de diabetes mellitus em $2014^{(7)}$. Possui o objetivo de verificar o nível de agenciamento de autocuidado, quanto à sua operacionalidade. É composta por 15 questões, e a graduação por escala Likert, variando de 1 (discordo totalmente) a 5 (concordo totalmente). Quatro questões se referem a aspectos negativos, tendo a necessidade de o escore ser invertido na análise dos dados. O escore varia entre 15 e 75 pontos, quanto maior pontuação maior capacidade de autocuidado operacionalizada ${ }^{(7)}$.

O KDQOL-SF foi desenvolvido pelo Working Group em 1997, e validado para o contexto brasileiro em $2005^{(16)}$, sendo um instrumento específico que avalia QVRS no contexto da DRC. Possui 80 itens, divididos em 19 dimensões analisadas separadamente. Não existe um valor único da avaliação global da QVRS, mas escores para cada dimensão. Essa análise possibilita identificar os reais problemas relacionados à saúde dos pacientes, e verificar o impacto na qualidade de vida. A pontuação final de cada dimensão varia de zero a 100 , sendo que a pontuação mais alta reflete melhor qualidade de vida ${ }^{(16)}$.

Os referidos instrumentos foram aplicados previamente à sessão de hemodiálise, ou na sua impossibilidade, nas duas primeiras horas de tratamento. Considerando a eventualidade de algum dos participantes apresentarem problemas visuais e/ou baixo nível instrucional, a aplicação dos instrumentos ocorreu por meio de entrevista individual com duração de 30 a $40 \mathrm{mim}$. Os dados foram coletados no período 
de junho a agosto de 2014. A aplicação do protocolo de avaliação foi realizada por duas estudantes com treinamento prévio específico.

Os dados foram digitados em planilha Microsoft Excel e transportados para a análise no software Statistical Package for the Social Sciences (SPSS para Windows), versão 22.0. Para análise descritiva dos dados, foram calculadas as médias, mínimas, máximas e desvio padrão, além da variação e frequência. Foi utilizado o alfa de Cronbach $(\alpha)$ para verificar a consistência interna da ASAS-R. O teste de KolmogorovSmirnov foi utilizado para verificar a aderência dos dados à normalidade e o Coeficiente de Correlação de Pearson para analisar a relação entre os escores da ASAS-R e os domínios do KDQOL-SF. Neste estudo, a magnitude das correlações foi classificada conforme proposição ${ }^{(17)}$ : fraca $(<0,3)$; moderada $(0,3$ a 0,59$)$, forte $(0,6$ a 0,9$)$ e perfeita $(1,0)^{(18)}$.

O desenvolvimento do estudo atendeu integralmente à Resolução 466/2012. E a pesquisa foi aprovada pelo Comitê de Ética em Pesquisa com Seres Humanos da Universidade Federal de São Carlos (Parecer no 509.241/2014).

\section{RESULTADOS}

Dos 100 participantes investigados, houve predomínio do sexo masculino (66,0\%), com média de idade de $53,2( \pm 14,72)$ anos, sendo que $60,0 \%$ estavam na faixa etária entre 22 a 59 anos e $40,0 \%$ tinham idade compreendida dos 60 a 79 anos. A grande maioria possuía parceiro fixo (74,0\%). O tempo médio de escolaridade foi de $7,41( \pm 4,33)$ anos, com prevalência do ensino fundamental incompleto $(46,0 \%)$. A média da renda mensal foi de $R \$ 869,73( \pm 1085,83)$ (Tabela 1$)$.

Tabela 1: Distribuição das variáveis sociodemográficas dos pacientes com DRC em hemodiálise. São Carlos, SP, Brasil, 2016.

\begin{tabular}{|c|c|c|c|c|c|c|}
\hline Variável & Média ( $\pm D p)$ & Mediana & Variação Observada & Distribuição em categorias & $\mathbf{N}$ & $\%$ \\
\hline \multirow{2}{*}{ Gênero } & \multirow{2}{*}{--} & \multirow{2}{*}{--} & \multirow{2}{*}{-- } & Masculino & 66 & 66,0 \\
\hline & & & & Feminino & 34 & 34,0 \\
\hline \multirow{2}{*}{ Idade (em anos) } & \multirow{2}{*}{$53,25( \pm 14,72)$} & \multirow{2}{*}{55} & \multirow{2}{*}{$22-79$} & 22 a 59 & 60 & 60,0 \\
\hline & & & & 60 a 79 & 40 & 40,0 \\
\hline \multirow{2}{*}{ Situação Conjugal } & \multirow{2}{*}{--} & \multirow{2}{*}{--} & \multirow{2}{*}{--} & Com parceiro & 74 & 74,0 \\
\hline & & & & Sem parceiro & 26 & 26,0 \\
\hline \multirow{4}{*}{ Escolaridade } & \multirow{4}{*}{$7,41( \pm 4,33)$} & \multirow{4}{*}{8} & \multirow{4}{*}{--} & Analfabeto & 3 & 3,0 \\
\hline & & & & 1 a 4 anos & 46 & 46,0 \\
\hline & & & & 5 a 9 anos & 21 & 21,0 \\
\hline & & & & 10 anos ou mais & 30 & 30,0 \\
\hline \multirow{3}{*}{ Renda per capita mensal $\mathrm{R} \$$} & \multirow{3}{*}{$869,73( \pm 1085,83)$} & \multirow{3}{*}{724} & \multirow{3}{*}{$90-10.000,00$} & Até 1 SM & 58 & 58,0 \\
\hline & & & & 1,1 à 3 SM & 38 & 38,0 \\
\hline & & & & 3,1 ou mais SM & 4 & 4,0 \\
\hline
\end{tabular}

Os resultados da avaliação da capacidade para o autocuidado estão apresentados na Tabela 2. A média do escore da escala total foi de $60,64( \pm 8,24)$. Entre os fatores avaliados pela ASAS-R, o fator $1-$ Ter capacidade para o autocuidado, apresentou uma média de 25,25 $( \pm 3,45)$, o fator 2 - Desenvolvimento para a capacidade do autocuidado, mostrou uma média de $20,62( \pm 3,03)$ e o fator 3 - Falta de capacidade para o autocuidado, apresentou média de 11,45 ( $\pm 2,35)$ (Tabela 2 ). 
Tabela 2: Estatística descritiva dos escores da ASAS-R aplicada aos 100 pacientes com DRC em hemodiálise. São Carlos, SP, Brasil, 2016.

\begin{tabular}{ccccc}
\hline Variável & Média (Dp \pm$)$ & Mediana & Variação & Alfa de Cronbach \\
\hline ASAS-R total & $60,64( \pm 8,24)$ & 59 & $35-75$ & 0,86 \\
Tendo Poder & $25,25( \pm 3,45)$ & 24 & $14-30$ & 0,85 \\
Desenvolvendo Poder & $20,62( \pm 3,03)$ & 20 & $10-25$ & 0,68 \\
Faltando Poder & $11,45( \pm 2,35)$ & 11 & 0,76 \\
\hline
\end{tabular}

A correlação entre a capacidade para o autocuidado e a QVRS das pessoas com DRC em tratamento hemodialítico foi positiva, de moderada magnitude, com significância estatística entre a ASAS-R e o KDQOLSF, nos domínios: sintomas/problemas ( $r=0,328 ; p=0,001)$, função cognitiva $(r=0,401 ; p<0,001)$, funcionamento físico ( $r=0,375 p<0,001)$, bem-estar emocional $(r=0,313 ; p=0,002)$ e energia/fadiga $(r=0,333$; $p=0,001)$ (Tabela 3).

Observa-se na Tabela 3 correlações fracas, estatisticamente significantes entre a ASAS-R e os seguintes domínios do KDQOL-SF: Efeitos da Doença Renal $(r=0,274 ; p=0,006)$, Qualidade da interação social $(r=0,268$; $p=0,007)$, Sono ( $r=0,087 ; p=0,562)$, Dor $(r=0,212 ; p=0,034)$, Saúde Geral $(r=0,260 ; p=0,009)$ e Função Social $(r=0,236 ; p=0,018)$.

Tabela 3: Coeficiente de Correlação de Pearson entre os escores da ASAS-R e dos domínios do KDQOL-SF aplicados aos pacientes com DRC em hemodiálise. São Carlos, SP, Brasil, 2016.

\begin{tabular}{ccc}
\hline Dimensões & $\mathbf{r}$ & ASAS-R \\
\cline { 2 - 3 } Sintomas/problemas & $\mathbf{0 , 3 2 8}$ & $\mathbf{0 , 0 0 1}$ \\
Efeitos da Doença Renal & $\mathbf{0 , 2 7 4}$ & $\mathbf{0 , 0 0 6}$ \\
Carga da Doença Renal & 0,176 & 0,08 \\
Situação de trabalho & 0,018 & 0,861 \\
Função cognitiva & $\mathbf{0 , 4 0 1}$ & $<\mathbf{0 , 0 0 1}$ \\
Qualidade da interação social & $\mathbf{0 , 2 6 8}$ & $\mathbf{0 , 0 0 7}$ \\
Função sexual & 0,087 & 0,562 \\
Sono & $\mathbf{0 , 1 9 7}$ & $\mathbf{0 , 0 4 9}$ \\
Suporte social & 0,144 & 0,154 \\
Incentivo de equipe de diálise & 0,028 & 0,78 \\
Satisfação do paciente & 0,025 & 0,802 \\
Funcionamento Físico & $\mathbf{0 , 3 7 5}$ & $\mathbf{0 , 0 0 1}$ \\
Função Física & 0,115 & 0,255 \\
Dor & $\mathbf{0 , 2 1 2}$ & $\mathbf{0 , 0 3 4}$ \\
Saúde Geral & $\mathbf{0 , 2 6}$ & $\mathbf{0 , 0 0 9}$ \\
Bem Estar Emocional & $\mathbf{0 , 3 1 3}$ & $\mathbf{0 , 0 0 2}$ \\
Função Emocional & $\mathbf{0 , 1 2 4}$ & 0,219 \\
Função Social & $\mathbf{0 , 2 3 6}$ & $\mathbf{0 , 0 1 8}$ \\
Energia/Fadiga & $\mathbf{0 , 3 3 3}$ & $\mathbf{0 , 0 0 1}$ \\
\hline
\end{tabular}

\section{DISCUSSÃO}

A amostra do estudo pode ser descrita predominantemente por homens, adultos jovens e com parceiro fixo e ensino fundamental incompleto. Os aspectos esboçados corroboram com os achados em estudos com paciente com DRC em diálise ${ }^{(19-20)}$.

Com relação à capacidade para o autocuidado avaliada por meio da ASAS-R, o escore médio obtido foi de $60,64( \pm 8,24)$. Vale reiterar que a pontuação pode variar de 15 a 75 pontos, quanto maior, maior também 
a capacidade para o autocuidado.

Na busca literária por estudos que utilizaram a ASAS-R para mensurar o nível de autocuidado, foi encontrada a pesquisa ${ }^{(6)}$ que adaptou, traduziu e validou a escala ASAS-R para o uso no contexto brasileiro, em pacientes com diabetes mellitus tipo 2, realizado em Ribeirão Preto/SP. Dentre os resultados citados, obtiveram o escore médio de $56,8( \pm 0,48)$ na aplicação da referida escala, indicando uma boa capacidade para o autocuidado, corroborando com os dados dessa pesquisa, embora em outra população.

Em outra pesquisa ${ }^{(21)}$ com 627 indivíduos de 17 estados brasileiros, verificou-se que o nível de agenciamento para a capacidade de autocuidado, avaliada por meio da ASAS-R, apresentou correlação significativa com a idade, com o nível educacional e com a renda. Em uma parcela da amostra ( $n=134$ ), composta por pacientes com doença crônica, identificou-se que quanto maior o nível de agenciamento para a capacidade de autocuidado, menor era o impacto negativo da doença no cotidiano dos sujeitos. Não foram fornecidos os valores médios do escore total da ASAS-R, uma vez que, a pesquisa tinha por objetivos apresentar as propriedades psicométricas da versão brasileira da ASAS-R, o que não permite a comparação dos dados com a presente pesquisa.

Vale salientar que não foram encontradas pesquisas que utilizaram a escala ASAS-R em pacientes com DRC em diálise, entretanto existem alguns estudos que abordam a temática referente ao autocuidado ${ }^{(22-24)}$.

Em um estudo ${ }^{(22)}$ realizado em Bogotá, com o objetivo de avaliar o nível de operacionalidade para a capacidade do autocuidado, por meio da ASAS-A (versão original da ASAS-R) em indivíduos em diálise peritoneal, encontrou-se uma média de 78,7 da ASAS-A total. Dos participantes, 72,4\% classificaram-se na faixa de alta capacidade para o autocuidado, demonstrando semelhança com os dados desta pesquisa, a qual apresentou boa capacidade para o autocuidado.

No estudo realizado ${ }^{(23)}$ no Rio Grande do Sul, no qual, almejou-se conhecer as práticas de autocuidado utilizadas por pessoas com DRC submetidos à diálise peritoneal, por meio de um roteiro com questões norteadoras, como: “O que o(a) senhor(a) faz para se cuidar?" “Quais os cuidados que o(a) senhor(a) tem com a ingestão de alimentos e de líquidos?" "Como é seu sono e repouso" "Como realiza o procedimento de diálise?". Identificaram que os nove participantes realizavam ações de autocuidado, tanto relacionadas à doença (alimentação, ingesta hídrica, sono, repouso, lazer e autoestima), quanto relacionadas à diálise (cateter e procedimento). Além disso, concluíram que os pacientes recebiam orientações e eram estimulados a cada consulta de enfermagem, a exercerem ações para seus próprios cuidados. Embora a pesquisa ${ }^{(23)}$ não utilizasse a escala deste estudo, permitindo a comparação dos dados, pode-se considerar que os pacientes também têm realizado ações de autocuidado, já que os resultados indicam que a grande maioria possui capacidade para o autocuidado satisfatória.

Estudo $^{(24)}$ com 214 pacientes de diversos Centros de Diálise em São José do Rio Preto-SP, buscou verificar a associação da independência funcional, por meio do instrumento de Medida de Independência Funcional (MIF) e da capacidade do autocuidado, utilizando a escala ASAS-A, com as variáveis sociodemográficas e clínicas em pacientes com DRC em tratamento hemodialítico, identificando resultados 
satisfatórios de independência funcional (média $=118,38$ ) e de capacidade para o autocuidado (média= 94,53), corroborando com a presente pesquisa. Além disso, o referido estudo permitiu identificar que as variáveis sexo, idade, comorbidades e complicações relacionadas ao tratamento hemodialítico são fatores que prejudicaram a independência funcional e a capacidade do autocuidado e, à medida que a idade aumenta, diminuindo a independência funcional e a capacidade para o autocuidado, além de que maiores números de comorbidades se relacionam com menor independência e menor capacidade para o autocuidado.

Na busca por estudos que abordassem a relação do autocuidado com a QVRS, foi encontrado apenas um $^{(25)}$, que avaliou 43 sujeitos através de um formulário para levantamento das necessidades de autocuidado e o KDQOL-SF. Os resultados indicaram as necessidades de orientação de enfermagem para o autocuidado sobre problemas de saúde física relacionado com terapia nutricional, ingestão de líquidos, complicações da hemodiálise, anticoagulação e prática de atividade física. Relacionado à função emocional verificou-se associação a grupos e a atividades de lazer e para a função física e sobrecarga da doença com a prática de atividade física. Os autores ainda afirmaram que à medida que o paciente alcança autonomia e independência nas ações de autocuidado, as dimensões afetadas pelo KDQOL-SF, como problemas emocionais, suporte social, efeitos da doença renal, melhoram.

Na grande maioria dos estudos ${ }^{(21-25)}$, os pacientes sinalizam e sugerem a relevância em se exercer as ações de autocuidado, já que a patologia por si só, demanda uma maior atenção, restrições e conhecimentos, fazendo com que os pacientes com DRC desempenhem e realizem mais ações em seu dia a dia para o cuidado e benefício próprio, a fim de manter a saúde, o bem-estar e o controle do seu tratamento, buscando qualidade de vida.

\section{CONCLUSÃO}

Conclui-se que os indivíduos com doença renal crônica em tratamento hemodiálitico apresentam um bom nível de agenciamento para a capacidade de autocuidado. Ademais, existe correlação positiva, diretamente proporcional, entre o autocuidado e a QVRS.

Espera-se que este estudo contribua positivamente para a construção de estratégias para a manutenção, implementação e assistência prestada aos pacientes renais crônicos, uma vez que as alterações em alguns domínios da QVRS identificadas neste estudo, tais como: sintomas/problemas, funções cognitivas, qualidade da interação social, sono, função social, entre outras, podem ser trabalhadas tanto pelos próprios pacientes, quanto pela família e a equipe.

Algumas estratégias poderiam contribuir para melhorias dos domínios afetados na qualidade de vida dos pacientes, como atividades lúdicas durante as sessões de hemodiálise, a fim de contribuir para a melhoria das funções cognitivas, o fortalecimento da qualidade da interação social no próprio ambiente de tratamento com o envolvimento de toda a equipe, orientações acerca da qualidade do sono, informações básicas aos pacientes entre outras estratégias, minimizam o impacto da doença, melhoram as ações de autocuidado, 
bem como contribuem com a melhoria da qualidade de vida.

A limitação desta pesquisa diz respeito ao desenho transversal do estudo que impossibilita a identificação da precedência temporal dos fatores estudados, comprometendo as evidências de relações de causa e efeito.

\section{Agradecimentos}

À Fundação de Amparo à Pesquisa do Estado de São Paulo (FAPESP) pelo apoio financeiro (Processo $\mathrm{n}$. 2014/07033-4)

\section{REFERÊNCIAS}

1. Organização Mundial da Saúde. Cuidados inovadores para condições crônicas: componentes estruturais de ação. Relatório Mundial. Brasília: OMS, 2003. 105 p.

2. Kirsztajn GM, Filho NS, Draibe SA, Netto MVP, Thomé FS, Souza E, Bastos MG. Fast Reading of the KDIGO 2012: Guidelines for evaluation and management of chronic kidney disease in clinical practice. J Bras Nefrol. 2014; 36(1):6373

3. Bertolin D, Haas V, Kusumota L, Pace A. Associação entre os modos de enfrentamento e as variáveis sociodemográficas de pessoas em hemodiálise crônica. Rev. Esc. Enferm. USP. 2011; 45(5) :1070-1076.

4. Ferreira RC, Silva Filho CR. A qualidade de vida dos pacientes renais crônicas em hemodiálise na Região de Marília, São Paulo. Jornal Brasileiro de Nefrologia 2011; 33(2).

5. Santos I, Rocha R, Berardinelli L. Necessidades de orientação de enfermagem para o autocuidado de clientes em terapia de hemodiálise. Rev. Bras. Enferm. 2011; 64(2): 335-42.

6. David HC et al. Análise da compreensão do autocuidado dos pacientes renais crônicos em tratamento hemodialítico e a influência da ansiedade e depressão em clínica especializada de Campo Grande-MS. Ensaios e Ciência: Ciências Biológicas, Agrárias e de Saúde. 2013; 17(5): 63-74.

7. Stacciarini T, Pace A. Tradução, adaptação e validação de uma escala para o autocuidado de portadores de diabetes mellitus tipo 2 em uso de insulina. Acta Paul Enferm. 2014; 27(3): 221-229.

8. Orem, DE. Nursing: concepts of practice. $6^{\text {th }}$ ed. St Louis: Mosby, 2001.

9. Pacheco GS, Santos I, Bregman R. Clientes com doença renal crônica: avaliação de enfermagem sobre a competência para o autocuidado. Esc. Anna Nery R. Enferm. 2007; 11(1): 44-51.

10. Terra FS. Avaliação da qualidade de vida do paciente renal crônico submetido à hemodiálise e sua adesão ao tratamento farmacológico de uso diário. Rev Bras Clin Med. 2010; 8(2): 119-24.

11. Ferreira FS, Santos CB. Qualidade de Vida Relacionada à Saúde de Pacientes Diabéticos atendidos pela equipe saúde da família. Rev. Enferm. UERJ. 2009; 17(3): 406-411.

12. Trevisol DJ. Qualidade de vida e hipertensão arterial sistêmica: estudo de base populacional na cidade de Porto Alegre (RS). [Tese]. Porto Alegre (RS): Faculdade de Medicina da Universidade Federal do Rio Grande do Sul; 2010. 21p.

13. Silva IJ et al. Cuidado, autocuidado e cuidado de si: uma compreensão paradigmática para o cuidado da enfermagem. Rev. Esc. Enferm. 2009; 43(3): 697-703.

14. Evers GCM et al. The appraisal of self-care agency's ASA-SCALE: research program to test reliability and validity. In: Proceedings of the International Nursing Research Conference "New Frontiers in Nursing Research". Edmond, Canada: University of Alberta, 1986. 130 p.

15. Sousa VD et al. Reliability validity and factor structure of the Appraisal of Self Care Agency Scale Revised (ASAS-R). J.Eval. Clin. Pract. 2010; 16(6): 1031-1040.

16. Duarte PS, Miyazaki MCOS, Ciconelli RM, Sesso R. Cultural adaptation and validation of the Kidney Disease and Quality of Life - Short Form (KDQOL - SF 1.3) in Brazil. Brazilian Journal of Medical and Biological Research. 2005; 38(2): 261-270. 
17. Levin J, Fox JA. Estatística para ciências humanas. $9^{\text {th }}$ ed. São Paulo: Prentice-Hall. 2004.

18. Hair JF, Black WC, Babin BJ, Anderson RE, Tatham RL. Análise multivariada de dados. Porto Alegre, RS: ArtMed. 2009.

19. Orlandi FS, Pepino BG, Pavarini SCl, Santos DA, Mendiondo MS. Avaliação do nível de esperança de vida de idosos renais crônicos em hemodiálise. Rev. Esc Enferm USP. 2012;46: 900-5.

20. Ottaviani AC, Souza EN, Drago NC, Mendiondo MS, Pavarini SCI, Orlandi FS. Esperança e Espiritualidade de adultos e idosos renais crônicos em hemodiálise: um estudo correlacional. Rev. Latino-Am. Enfermagem. 2014; 22(2): $248-54$. 21. Damásio, B, Koller S. The Appraisal of Self-Care Agency Scale - Revised (ASAS-R): adaptation and construct validity in the Brazilian context. Cad. Saúde Pública. 2013; 29(10): 2071-2082.

22. Algarra, AJC. Análisis de la capacidad de autocuidado en pacientes en diálisis peritoneal. Enferm. Nefrol. 2015; 18(1): 31-49.

23. Calderan C, Torres AAP, Zillmer JGV, Schwartz E, Silva DGV. Práticas de autocuidado de pessoas com insuficiência renal crônica submetidas à diálise peritoneal ambulatorial contínua. Rev. Pesq.: Cuid. Fundam. Online. 2013; 5(1): 394402.

24. Oller GASAO, Ribeiro RCHM, Travagim DAS, Batista MA, Marques S, Kusumota L. Independência funcional em pacientes com doença renal crônica em tratamento hemodialítico. Rev. Latino-AM. Enfermagem. 2012; 20(6). 25. Santos I, Rocha RPF, Berardinelli LMM. Qualidade de vida de clientes em hemodiálise e necessidades de orientação de enfermagem para o autocuidado. Esc. Anna Nery. 2011; 15(1). 